\title{
Efficacy of interspace between the popliteal artery and the capsule of the posterior knee (iPACK) block versus periarticular local infiltration analgesia after unilateral total knee arthroplasty
}

\author{
Prospective randomized control trial
}

Abdul Sattar Narejo, FCPS, FCAI, Fatima Abdulwahab, MD, Mansoor Aqil, FCPS, Abdullah T. Alsubaie, MD, Hassan Y. Hazazy, MD, Tariq Alzahrani, MD, Abdulaziz Aljurayyan, MD, FRCSc, Ahmed Thallaj, MD, EDRA.

\begin{abstract}

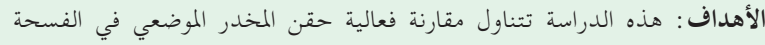

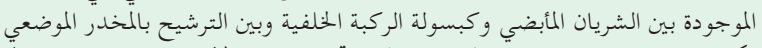

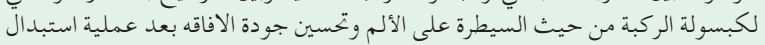

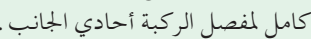

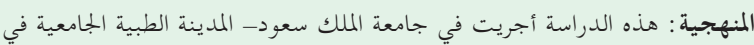

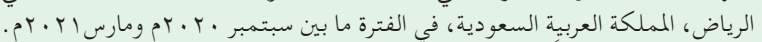

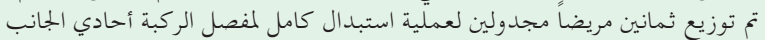

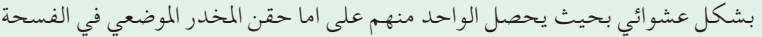

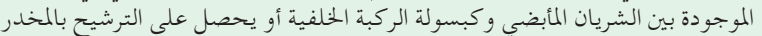

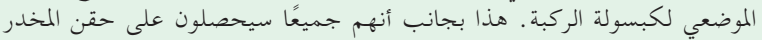

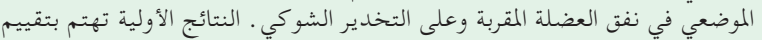

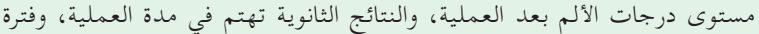

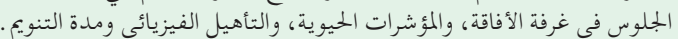

النتائج: مستوى الألم مع الحركة في المجموعة الحاصلة على الحقن في الفستحة

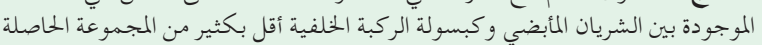

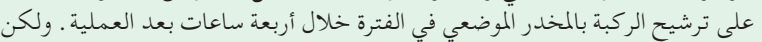

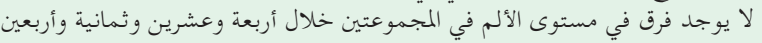

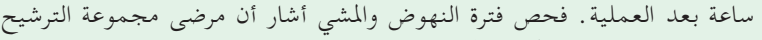

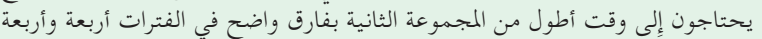

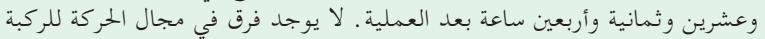
في كلا المجموعتين.

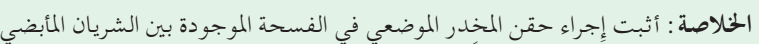

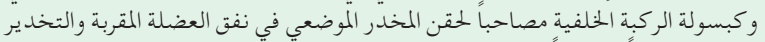

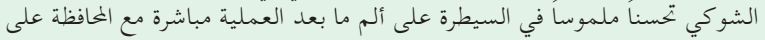

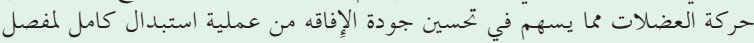

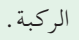

Objectives: To compare the efficacy of interspace between the popliteal artery and the capsule of the posterior knee (iPACK) block with periarticular local infiltration analgesia (LIA) to assess postoperative pain control and enhanced recovery after total knee arthroplasty (TKA).

Methods: This research was carried out at King Saud University Medical City, Riyadh, Saudi Arabia, from September 2020 to March 2021. Eighty Patients scheduled for elective unilateral TKA were randomized to receive either iPACK or periarticular LIA along with adductor canal block under spinal anesthesia. The primary outcome was postoperative pain score, and secondary outcomes included physical rehabilitation, duration of surgery, length of post-anesthesia care unit stay, hemodynamics, and length of hospital stay.

Results: The pain score during activity in iPACK group was significantly lower compared to LIA group at 4 hours postoperatively, but no significant difference was observed at 24 or 48 hours. The timed up and go test took significantly longer for patients in LIA group at 4 , 24 , and 48 hours compared to those in iPACK group. No significant differences in knee range of motion were observed between the 2 groups at any point.

Conclusion: Based on our findings, iPACK block is an effective technique in reducing pain in the immediate postoperative period without affecting motor function, resulting in enhanced recovery following primary TKA.

Keywords: iPACK, adductor canal block, local infiltration analgesia, total knee arthroplasty

Saudi Med J 2021; Vol. 42 (10): 1065-1071

doi: 10.15537/smj.2021.42.10.20210504

From the Department of Anesthesiology (Narejo, Abdulwahab, Aqil, Alsubaie, Alzahrani, Thallaj); from the Department of Orthopedic Surgery (Aljurayyan), King Saud University Medical City; and from the Department of Anesthesiology (Hazazy), Prince Sultan Military Medical City, Riyadh, Kingdom of Saudi Arabia.

Received 23rd June 2021. Accepted 25th August 2021.

Address correspondence and reprint request to: Dr. Abdul Sattar Narejo, Consultant, Department of Anesthesiology, King Saud University Medical City, Riyadh, Kingdom of Saudi Arabia. E-mail: narejo27@hotmail.com

ORCID ID: https://orcid.org/0000-0001-8016-1370 
$\mathrm{O}$ ptimal pain control and safe postoperative early mobilization are the main objectives of enhanced recovery after total knee arthroplasty (TKA). ${ }^{1,2}$ The trend of postoperative analgesia after TKA has been shifted from epidural and femoral nerve blocks (FNB) to motor-sparing sensory nerve blocks. Femoral nerve block is an effective technique to control anteromedial pain and reduce postoperative opioid consumption post TKA but results in quadriceps muscle weakness, making physical rehabilitation unsatisfactory. ${ }^{3-5}$ Alternatively, blocking the saphenous nerve in the adductor canal provides comparable anteromedial pain relief and preserves quadriceps strength comparing to FNB after TKA. ${ }^{6-8}$

Human knee innervation is complex. ${ }^{9}$ The posterior knee joint is innervated by popliteal plexus, formed by the tibial and posterior branches of the obturator nerves. ${ }^{10}$ The efficacy of blocking these terminal sensory branches at the posterior knee capsule is under investigation. Pain that arises from the posterior knee after TKA may be ameliorated by ultrasound-guided local anesthetic infiltration of the interspace between the popliteal artery and the capsule of the posterior knee (iPACK). ${ }^{11,12}$ Since the first description of iPACK block by Sinha (unpublished observation)extensive research has been carried out to assess its efficacy. ${ }^{13,14}$ The advantages of iPACK compare to other modalities for posterior knee pain control are its motor-sparing analgesic effectiveness, reduced postoperative opioid consumption, and improved functional measures. ${ }^{15}$ Unwanted complications during iPACK block include the risk of common peroneal nerve block, intravascular injection, or vascular injury to the nearby popliteal vessels.

In contrast to the local anesthetic infiltration of iPACK, intraoperative periarticular local infiltration analgesia (LIA) is a standard analgesic option for acute pain management after TKA. However, LIA block is performed by orthopedic surgeons on landmark technique. Its efficacy depends on the technique and the analgesic drugs regime used, but there is no consensus. ${ }^{16}$ Therefore, provided the potential advantage of motorsparing and may be comparable analgesia of iPACK, this study aimed to compare iPACK block with routine periarticular LIA block when combined with

Disclosure. Authors have no conflict of interests, and the work was not supported or funded by any drug company. adductor canal block (ACB) under spinal anesthesia. We hypothesize that iPACK is an effective technique to provide adequate analgesia after TKA.

Methods. We performed a prospective randomized controlled trial among patients who underwent elective, primary unilateral TKA under spinal anesthesia. The trial was registered at clinicaltrials.gov \#NCT04565093. After obtaining Institutional Review Board approval (No. E-20-4819, from King Khalid University Hospital, Riyadh, Saudi Arabia), this study was carried out from September 2020 to March 2021. The inclusion criteria were having an American Society of Anesthesiologists (ASA) classification of I-III, being $\geq 18$ years old, and having a body mass index (BMI) of $\leq 50 \mathrm{~kg} / \mathrm{m}^{2}$. Patients were excluded if they had a history of rheumatoid arthritis, renal impairment, liver disease, had undergone prior back surgery, were on any anticoagulant, or had any other contraindication for spinal anesthesia.

All patients were informed and consented to receive either iPACK block + ACB (iPACK group; $n=40$ ) or periarticular LIA block + ACB (LIA group; $n=40$ ) after spinal anesthesia. On arrival to the operation room, all eligible participants had an intravenous cannula in situ and monitors according to ASA guidelines. Under complete aseptic technique, spinal anesthesia was performed with a pencil-point 27-gauge (Whitacre) needle at lumbar space L3-L4 or L4-L5, with bupivacaine heavy $(0.5 \%)$ and fentanyl $(15 \mu \mathrm{g})$, for a total volume of $3 \mathrm{~mL}$.

The randomization scheme was generated using an online software (www.randomizer.org). Random allocation to either the iPACK or LIA group was indicated in a sealed, opaque envelope organized and opened in the block area by the anesthesia nurse. All participants and postoperative outcome assessors (physiotherapists) were blinded to the group allocation. The anesthesiologist responsible for intraoperative care and the operating surgeon were aware of the randomization.

Description of peripheral nerve block techniques. Infiltration of the interspace between the popliteal artery and the capsule of the posterior knee and ACB were performed using a complete aseptic technique and under ultrasound guidance by consultants anesthesiologists or regional anesthesia fellows approximately within 10-15 minutes. Four senior orthopedic consultants performed the periarticular LIA block under complete aseptic measures using a technique based on landmarks approximately within 5-10 minutes.

Adductor canal block. A linear transducer (SonoSite 15-6 MHz) was placed anteriorly distal to mid-thigh 
then slide medially to locate the femoral artery under the sartorius muscle. The needle (Pajunk, Germany, $80-100 \mathrm{~mm}$ ) was inserted in-plane in a lateral-to-medial orientation and advanced further to penetrate the vastoadductor membrane into the adductor canal toward the saphenous nerve if visible. After careful aspiration, 1-2 mL of normal saline was injected anterior and lateral to the femoral artery to confirm the proper injection site. The needle was redirected if the spread of normal saline was not evident. A total of $20 \mathrm{~mL}$ of bupivacaine $(0.25 \%)$ was injected into the adductor canal around the femoral artery. ${ }^{17}$

Interspace between the popliteal artery and the capsule of the posterior knee block. A curvilinear transducer (SonoSite 5-2 MHz) was placed on the popliteal crease to visualize the space between the popliteal artery and femoral condyles. The needle (Pajunk, Germany, 80-100 mm) was inserted in-plane from the transducer's anterior end in a medial-to-lateral trajectory. With the needle tip resting $2 \mathrm{~cm}$ beyond the lateral border of the artery, $20 \mathrm{~mL}$ of bupivacaine $(0.25 \%)$ was injected after a negative blood aspiration to infiltrate the tissue space in divided doses as the needle was withdrawn. ${ }^{11}$

Periarticular LIA block. A mixture of $20 \mathrm{ml}$ of bupivacaine $(0.5 \%), 100 \mu \mathrm{g}$ epinephrine, $\pm 10 \mathrm{mg}$ of Morphine, $\pm 8 \mathrm{mg}$ of lornoxicam, was diluted in $40 \mathrm{~mL}$ of normal saline. The posterior capsule was infiltrated with the mixture solution prior to implantation. After implantation, the rest of the solution was injected into the retinacular flaps, surrounding muscles, and subcutaneous tissues. ${ }^{18}$

Postoperative pain control. All participants were prescribed intravenous morphine $2 \mathrm{mg}$ bolus, up to a maximum of $10 \mathrm{mg}$, if indicated in the Post-Anesthesia Care Unit (PACU). The multimodal analgesic regimen for the ward included $8 \mathrm{mg}$ of intravenous lornoxicam every 12 hours and $1 \mathrm{gm}$ of paracetamol every 8 hours if indicated for postoperative days $0-1$. After 24 hours, $200 \mathrm{mg}$ of celecoxib was given orally every 12 hours, as well as $20 \mathrm{mg}$ of oxycodone orally every 12 hours and $75 \mathrm{mg}$ of pregabalin orally every 12 hours; in addition, $1 \mathrm{gm}$ of paracetamol, taken orally every 6 hours, was prescribed for breakthrough pain.

The primary outcome was the severity of pain assessed postoperatively at 4, 24, and 48 hours by 3 physiotherapists blinded to group allocation. Physical rehabilitation was evaluated by measuring the active-assisted knee range of motion $(\mathrm{ROM})^{19}$ using a goniometer (Sammons Preston-Rolyan \#7514, Bolingbrook, IL, USA), and a timed up and go (TUG) test used to assess postoperative ambulation. Timed up and go test uses the time a person takes to rise from an armchair, walk 3 meters, turn around, walk back to the chair, and sit down. ${ }^{20}$ All patients were instructed preoperatively on how to report postoperative pain on a 10-point numerical rating scale (NRS), where 0 is no pain and 10 is the worst pain imaginable. The secondary outcomes were physical rehabilitation progress, duration of surgery, length of PACU stay, postoperative hemodynamics, length of hospital stay (LOS), and any adverse effects like vascular injury, common peroneal nerve block, intravascular injection/ local anesthetic systemic toxicity or any other iPACK- or ACB-related complications.

Statistical analysis. A previous trial found that the mean \pm standard deviation of pain score at 24 hours post TKA to be $4.9 \pm 2.7$ points, and post iPACK to be 2.5 points. Assuming $80 \%$ power at a 2 -sided number of 0.05 , we determined that a sample size of 40 patients per group total of 80 would be essential. ${ }^{12,14}$ Statistical analysis was performed using GraphPad Prism version 8.4.3 for Windows (GraphPad Software, La Jolla, CA, USA). Where appropriate, group means with a standard error of the mean (SEM) and sample size were reported. For all tests, differences between the groups were reported as exact $p$-values. Differences were considered statistically significant at an alpha level of $<0.05$. An unpaired student's t-test was used to calculate group differences between parametric data. Chi-square test was used to calculate group differences between non-parametric and categorical data.

Results. During recruitment, 105 patients were assessed for eligibility. Twenty-five patients were excluded from the study who met the exclusion criteria. We randomly allocated 80 patients to either the iPACK or LIA group to assess postoperative pain after TKA (Figure 1). The demographic data was comparable between the 2 groups (Table 1). There were no statistically significant differences between the 2 groups in relation to the duration of surgery, length of PACU stay, mean arterial pressure, heart rate, or LOS in days (Table 2).

As shown in Figure 2, the pain score was significantly lower in the iPACK group compared to the LIA group at 4 hours postoperatively (3.32 versus 4.75$)$ $(p=0.0046)$. No significant difference was observed after that at 24 hours $(p=0.8253)$ or 48 hours $(p=0.4098)$ when comparing the 2 groups. The TUG test, in seconds, was significantly longer in the LIA group at 4,24 , and 48 hours compared to the iPACK group $(p=0.0004, p=0.0013$, and $p=0.0017)$, respectively; (Figure 3). No significant differences were observed 


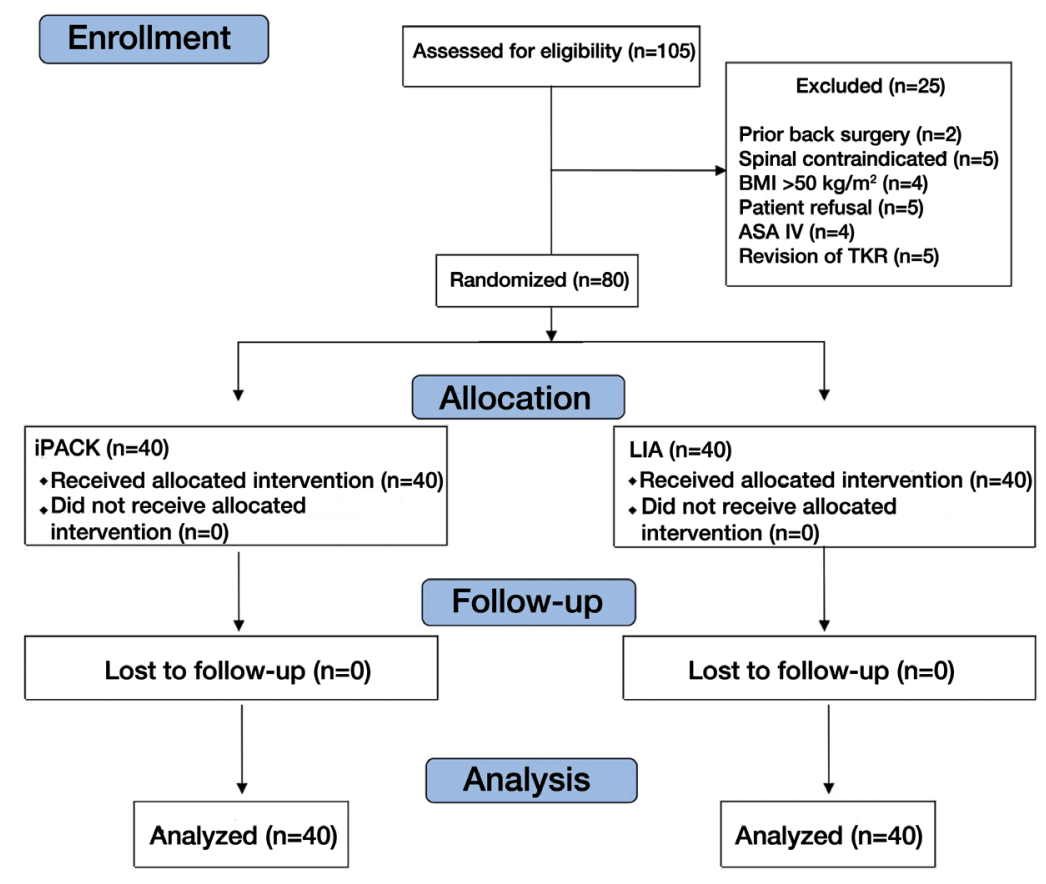

Figure 1 - Consolidated standards of reporting trial flow diagram. iPACK: interspace between the popliteal artery and the capsule of the posterior knee, LIA: local infiltration analgesia, GA: general anesthesia

Table 1 - Demographic data comparing iPACK and LIA groups.

\begin{tabular}{|c|c|c|c|c|}
\hline Characteristics & $\mathrm{iPACK}(\mathrm{n}=40)$ & LIA $(n=40)$ & $95 \% \mathrm{CI}$ & $P$-value \\
\hline \multirow[t]{2}{*}{ Age } & $64.28 \pm 1.15$ & $64.25 \pm 1.24$ & \multirow[t]{2}{*}{-3.388 to 3.338} & \multirow[t]{2}{*}{$0.9882^{*}$} \\
\hline & $63.00(59.00-69.75)$ & $63.50(58.00-69.75)$ & & \\
\hline \multirow[t]{2}{*}{$B M I$} & $33.46 \pm 0.97$ & $34.66 \pm 1.15$ & \multirow{2}{*}{-1.806 to 4.187} & \multirow{2}{*}{$0.4314^{*}$} \\
\hline & $33.53(29.15-36.08)$ & $34.62(27.65-39.85)$ & & \\
\hline \multicolumn{5}{|l|}{ Gender } \\
\hline Female & $30(75.0)$ & $33(83.0)$ & \multirow{2}{*}{ N/A } & \multirow{2}{*}{$>0.4123^{* *}$} \\
\hline Male & $10(25.0)$ & $7(18.0)$ & & \\
\hline \multicolumn{5}{|l|}{$A S A$} \\
\hline II & $35(87.5)$ & $35(87.5)$ & \multirow{2}{*}{ N/A } & \multirow{2}{*}{$>0.9999^{* *}$} \\
\hline III & $5(14.3)$ & $5(14.3)$ & & \\
\hline $\begin{array}{r}\text { Values are } \\
\text { statistically } \\
\text { iPACK } \\
\text { CI: confider }\end{array}$ & $\begin{array}{l}\text { ean } \pm \text { standard error of } \\
\text { alpha level of less than } \\
\text { veen the popliteal arter } \\
\text { apaired student's t-test }\end{array}$ & $\begin{array}{l}\text { an (SEM) and number } \\
\text { ASA: American Society } \\
\text { he capsule of the poster } \\
\text { d to analyze the data. * }\end{array}$ & $\begin{array}{l}\text { entages). Differenc } \\
\text { esthesiologists, BN } \\
\text { ee, LIA: local infil } \\
\text { quare test was use }\end{array}$ & $\begin{array}{l}\text { considered } \\
\text { mass index, } \\
\text { analgesia, } \\
\text { lyze the data. }\end{array}$ \\
\hline
\end{tabular}

between the 2 groups when comparing knee ROM at 4 hours $(p=0.7935), 24$ hours $(p=0.6979)$, or 48 hours $(p=0.3069)$ (Figure 4).

Discussion. This study was carried out to assess the efficacy of iPACK block compared with LIA block under spinal anesthesia and $\mathrm{ACB}$ in relation to postoperative pain control and early mobilization. We found that patients who received iPACK block reported mild pain in the early postoperative period only, while those in the LIA group reported moderate pain. This assessment was conducted immediately after the spinal anesthesia effect had worn off 4 hours after surgery. After that, the pain score was moderate in both groups on days one 
Table 2 - Intraoperative and postoperative patients characteristics comparing iPACK and LIA groups

\begin{tabular}{lcccc}
\hline Characteristics & iPACK & LIA & 95\% CI & $P$-value \\
\hline Duration of surgery (minutes) & $114.2 \pm 3.296$ & $122.1 \pm 3.118$ & -1.183 to 16.880 & 0.0876 \\
& $94.25-130.00$ & $107.00-137.50$ & & \\
PACU duration (minutes) & $61.0 \pm 3.114$ & $61.0 \pm 2.452$ & $-7.940-7.840$ & 0.9900 \\
& $48.25-65.00$ & $48.50-68.00$ & & \\
Mean arterial pressure (mmHg) & $89.3 \pm 1.757$ & $88.2 \pm 1.580$ & $-5.853-3.555$ & 0.6281 \\
& $81.25-95.00$ & $81.25-95.00$ & & \\
Heart rate (BPM) & $79.0 \pm 1.703$ & $76.1 \pm 1.374$ & $-7.282-1.432$ & 0.1853 \\
Length of hospital stay (days) & $72.00-86.50$ & $70.00-83.75$ & & \\
& $3.2 \pm 0.190$ & $3.2 \pm 0.275$ & $-0.6902-0.6402$ & 0.9406 \\
\hline
\end{tabular}

An unpaired student's t test was used to analyze the data between iPACK and LIA groups. Values are presented as mean \pm SEM. iPACK: interspace between the popliteal artery and the capsule of the posterior knee, LIA: local infiltration analgesia, BPM: beats per minute SEM: standard error of the mean, PACU: Post-Anesthesia Care Unit, CI: confidence interval

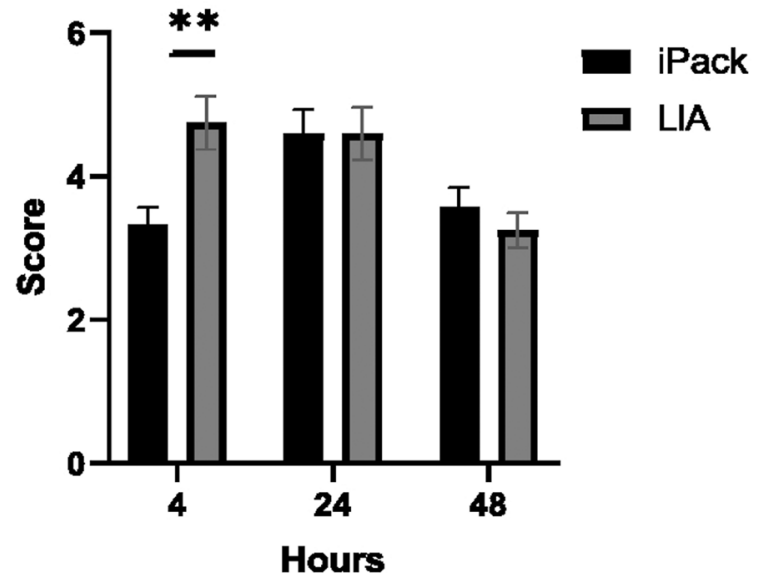

Figure 2 - Pain score during mobilization using Numerical Rating Scale (NRS $0-10$ ) where 0 is no pain and 10 is the worst pain imaginable at 4, 24, and 48 hours postoperatively comparing iPACK and LIA groups. ${ }^{*} p<0.05,{ }^{* *} p<0.01,{ }^{* * *} p<0.001$, iPACK: interspace between the popliteal artery and the capsule of the posterior knee, LIA: local infiltration analgesia

and 2. Similarly, there was no significant difference in analgesic consumption between the 2 groups. As the patients in the iPACK group had less pain, they had better mobility compared to those in the LIA group as early as 4 hours on the ward. The active-assisted knee ROM measurements, taken while the patients were still on the ward, were comparable between the 2 groups. Therefore, postoperative early rehabilitation was possible in the iPACK group due to better pain control

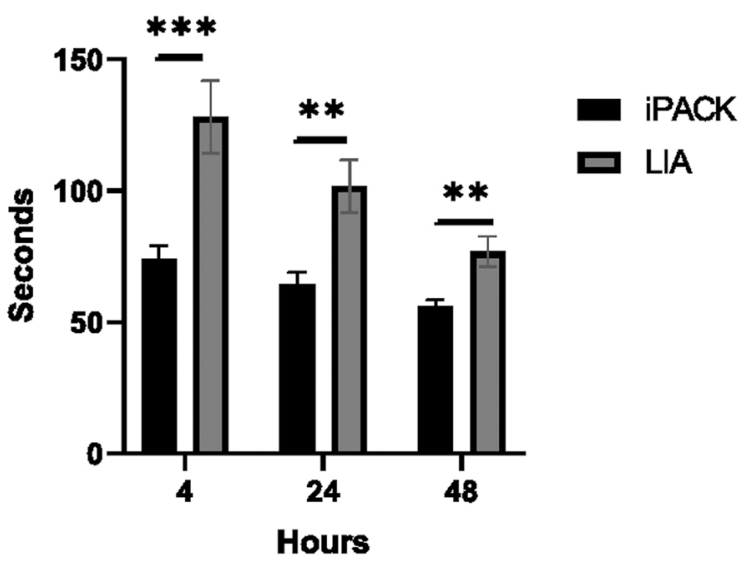

Figure 3 - Timed-Up-and-Go test in seconds at 4, 24, and 48 hours postoperatively comparing iPACK and LIA groups. ${ }^{*} p<0.05$, ${ }^{* *} p<0.01,{ }^{* * *} p<0.001$, iPACK: interspace between the popliteal artery and the capsule of the posterior knee, LIA: local infiltration analgesia

and the motor-sparing effect of the combination of iPACK block and ACB. These findings support those reported in a retrospective study conducted by Jung. ${ }^{21}$

Periarticular LIA block, first described by Kerr and Kohan. ${ }^{22}$ Although it is a blind landmark technique and there is no consensus on the optimal dose or medication regime for postoperative pain control, its analgesic effect is undoubted. A recent meta-analysis showed that local anesthetic infiltration is superior to epidural analgesia 


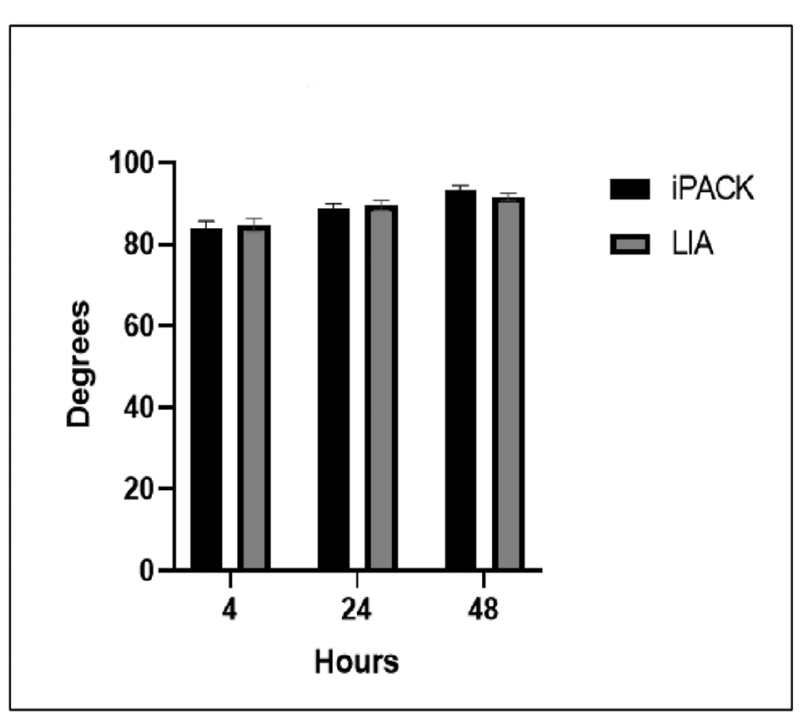

Figure 3 - Assisted-active knee range of motion (ROM) $0=$ full extension 135 =maximum flexion in degrees at 4, 24, and 48 hours postoperatively comparing iPACK and LIA groups. iPACK: interspace between the popliteal artery and the capsule of the posterior knee, LIA: local infiltration analgesia

for postoperative pain control after TKA. ${ }^{23}$ Perhaps the doses and techniques used for periarticular LIA were likely inconsistent between the different operating surgeons in our study. Another systemic review and meta-analysis investigated and did not support adding iPACK block to ACB in the presence of periarticular LIA due to lack of any additional benefit. ${ }^{24}$

The intra-articular excision of the knee evokes pain from structures being innervated by the popliteal plexus. Ultrasound-guided iPACK block mainly targets the terminal branches of the popliteal nerve plexus, which directly supply the posterior capsule of the knee. ${ }^{25}$ In 2 different studies, Kampitak et $\mathrm{al}^{2,25}$ discussed the optimal location of the motor-sparing effect of iPACK block and its related complications. In this study, we performed the iPACK block between the popliteal artery and the femoral condyles, just above the popliteal crease. None of our patients had vascular injury, common peroneal nerve block, intravascular injection/local anesthetic systemic toxicity, or other iPACK- or ACB-related complications.

Study limitations. First, the medication regimen (including dosages) and knee infiltration analgesia techniques used by different surgeons at our institute were not standardized. Second, patients' preoperative knee pain and medications were not recorded in this study. As such, further studies on a large number of patients are required to eliminate these limitations.
In conclusion, iPACK block is an effective technique in reducing pain in the immediate postoperative period without affecting motor function, resulting in enhanced recovery following primary TKA.

Acknowledgment. This work was supported by the College of Medicine Research Center, Deanship of Scientific Research, King Saud University, Riyadh, Kingdom of Saudi Arabia. The authors would like to thank Shatha Alsousy, Jameela Alshahrani, and Alhudrin Sahiol from the Department of Physiotherapy, King Saud University Medical City, Riyadh, Kingdom of Saudi Arabia for postoperative data collection. Moreover, we would like to thank Dr. Saara Mansoor, PhD. student, Dalhousie University, Halifax, Canada, for the statistical analysis of the data obtained. We would like to thank SCRIBENDI (https://www.scribendi.com) for English language editing.

\section{References}

1. Fransen BL, Hoozemans MJM, Argelo KDS, Keijser LCM, Burger BJ. Fast-track total knee arthroplasty improved clinical and functional outcome in the first 7 days after surgery: a randomized controlled pilot study with 5-year follow-up. Arch Orthop Trauma Surg 2018; 138: 1305-1316.

2. Kampitak W, Tanavalee A, Ngarmukos S, Tantavisut S. Motorsparing effect of iPACK (interspace between the popliteal artery and capsule of the posterior knee) block versus tibial nerve block after total knee arthroplasty: a randomized controlled trial. Reg Anesth Pain Med 2020; 45: 267-276.

3. Ortiz-Gómez JR, Perepérez-Candel M, Vázquez-Torres JM, Rodriguez-Del Río JM, Torrón-Abad B, Fornet-Ruiz I, et al. Postoperative analgesia for elective total knee arthroplasty under subarachnoid anesthesia with opioids: comparison between epidural, femoral block, and adductor canal block techniques (with and without perineural adjuvants). A prospective, randomized, clinical trial. Minerva Anestesiol 2017; 83: 50-58.

4. Sankineani SR, Reddy ARC, Eachempati KK, Jangale A, Gurava Reddy AV. Comparison of adductor canal block and IPACK block (interspace between the popliteal artery and the capsule of the posterior knee) with adductor canal block alone after total knee arthroplasty: a prospective control trial on pain and knee function in immediate postoperative period. Eur J Orthop Surg Traumatol 2018; 28: 1391-1395.

5. Koh IJ, Choi YJ, Kim MS, Koh HJ, Kang MS, In Y. Femoral nerve block versus adductor canal block for analgesia after total knee arthroplasty. Knee Surg Relat Res 2017; 29: 87-95.

6. Jiang X, Wang QQ, Wu CA, Tian W. Analgesic efficacy of adductor canal block in total knee arthroplasty: A meta-analysis and systematic review. Orthop Surg 2016; 8: 294-300.

7. Karkhur Y, Mahajan R, Kakralia A, Pandey AP, Kapoor MC. A comparative analysis of femoral nerve block with adductor canal block following total knee arthroplasty: A systematic literature review. J Anaesthesiol Clin Pharmacol 2018; 34: 433-438.

8. St Laurant DB, Peng P, Arango LG, Niazi AU, Chan VW, Agur $\mathrm{A}$, et al. The nerves of the adductor canal and the innervation of the knee: an anatomic study. Reg Anesth Pain Med 2016; 41 : 321-327.

9. Roberts SL, Stout A, Dreyfuss P. Review of knee joint innervation: implications for diagnostic blocks and radiofrequency Ablation. Pain Med 2020; 21: 922-938. 
10. Fonkoué L, Behets C, Kouassi JK, Coyette M, Detrembleur C, Thienpont E, et al. Distribution of sensory nerves supplying the knee joint capsule and implications for genicular blockade and radiofrequency ablation: an anatomical study. Surg Radiol Anat 2019; 41: 1461-1471.

11. Thobhani S, Scalercio L, Elliott CE, Nossaman BD, Thomas LC, Yuratich D, et al. Novel regional techniques for total knee arthroplasty promote reduced hospital length of stay: An analysis of 106 patients. Ochsner J 2017; 17: 233-238.

12. Kim DH, Beathe JC, Lin Y, YaDeau JT, Maalouf DB, Goytizolo $\mathrm{E}$, et al. Addition of infiltration between the popliteal artery and the capsule of the posterior knee and adductor canal block to periarticular injection enhances postoperative pain control in total knee arthroplasty: A randomized controlled trial. Anesth Analg 2019; 129: 526-535.

13. Tran J, Giron Arango L, Peng P, Sinha SK, Agur A, Chan V. Evaluation of the iPACK block injectate spread: a cadaveric study. Reg Anesth Pain Med 2019: rapm-2018-100355.

14. Albrecht E, Wegrzyn J, Dabetic A, El-Boghdadly K. The analgesic efficacy of iPACK after knee surgery: A systematic review and meta-analysis with trial sequential analysis. J Clin Anesth 2021; 72: 110305.

15. Ochroch J, Qi V, Badiola I, Grosh T, Cai L, Graff V, et al. Analgesic efficacy of adding the IPACK block to a multimodal analgesia protocol for primary total knee arthroplasty. Reg Anesth Pain Med 2020; 45: 799-804.

16. Seangleulur A, Vanasbodeekul P, Prapaitrakool S, Worathongchai $S$, Anothaisintawee T, McEvoy M, et al. The efficacy of local infiltration analgesia in the early postoperative period after total knee arthroplasty: A systematic review and meta-analysis. Eur J Anaesthesiol 2016; 33: 816-831.

17. Vora MU, Nicholas TA, Kassel CA, Grant SA. Adductor canal block for knee surgical procedures: review article. J Clin Anesth 2016; 35: 295-303.

18. O'Donnell R, Dolan J. Anaesthesia and analgesia for knee joint arthroplasty. BJA Educ 2018; 18: 8-15.
19. McGinnis RS, Patel S, Silva I, Mahadevan N, DiCristofaro S, Jortberg E, et al. Skin mounted accelerometer system for measuring knee range of motion. Annu Int Conf IEEE Eng Med Biol Soc 2016; 2016: 5298-5302.

20. Hsieh CY, Huang HY, Liu KC, Chen KH, Hsu SJ, Chan CT. Subtask segmentation of timed up and go test for mobility assessment of perioperative total knee arthroplasty. Sensors (Basel) 2020; 20: 6302.

21. Jung DW, Shon WY, Seo SS, Kim OG, Lee IS. Comparison of the postoperative analgesic effect for infiltration between the popliteal artery and the capsule of the posterior knee and that of periarticular multimodal drug injection in total knee arthroplasty: retrospective study in the immediate postoperative period. Knee Surg Relat Res 2020; 32: 1.

22. Kerr DR, Kohan L. Local infiltration analgesia: a technique for the control of acute postoperative pain following knee and hip surgery: a case study of 325 patients. Acta Orthop 2008; 79: 174-183.

23. Liu X, Zhang H, Zhang H, Guo M, Gao Y, Du C. Local infiltration vs epidural analgesia for postoperative pain control after total knee or hip arthroplasty: A meta-analysis of randomized controlled trials. Medicine (Baltimore) 2020 30; 99: e22674.

24. Hussain N, Brull R, Sheehy B, Dasu M, Weaver T, Abdallah FW. Does the addition of iPACK to adductor canal block in the presence or absence of periarticular local anesthetic infiltration improve analgesic and functional outcomes following total knee arthroplasty? A systematic review and meta-analysis. Reg Anesth Pain Med 2021; 46: 713-721.

25. Kampitak W, Tansatit T, Tanavalee A, Ngarmukos S. Optimal location of local anesthetic injection in the interspace between the popliteal artery and posterior capsule of the knee (iPACK) for posterior knee pain after total knee arthroplasty: an anatomical and clinical study. Korean J Anesthesiol 2019; 72 : 486-494. 